\title{
A gene that underwent adaptive evolution, LAC2 (LACCASE), in Populus euphratica improves drought tolerance by improving water transport capacity
}

\author{
Zhimin Niu', Guiting Li ${ }^{1}$, Hongyin $\mathrm{Hu}^{1}$, Jiaojiao Lv¹, Qiwei Zheng², Jianquan Liu ${ }^{1}$ and Dongshi Wan ${ }^{1}$
}

\begin{abstract}
Drought severely limits plant development and growth; accordingly, plants have evolved strategies to prevent water loss and adapt to water deficit conditions. However, experimental cases that corroborate these evolutionary processes are limited. The LACCASEs (LACS) family is involved in various plant development and growth processes, Here, we performed an evolutionary analysis of LACS from Populus euphratica and characterized the functions of LACS in Arabidopsis and poplar. The results showed that in PeuLACs, multiple gene duplications led to apparent functional redundancy as the result of various selective pressures. Among them, PeuLAC2 underwent strong positive selection. Heterologous expression analyses showed that the overexpression of PeuLAC2 alters the xylem structure of plants, including thickening the secondary cell wall (SCW) and increasing the fiber cell length and stem tensile strength. Altogether, these changes improve the water transport capacity of plants. The analysis of the physiological experimental results showed that PeULAC2-OE lines exhibited a stronger antioxidant response and greater drought tolerance than WT. Three genes screened by transcriptome analysis, NACO25, BG1, and UGT, that are associated with SCW synthesis and drought stress were all upregulated in the PeuLAC2-OE lines, implying that the overexpression of PeuLAC2 thickened the SCW, improved the water transport capacity of the plant, and further enhanced its drought tolerance. Our study highlights that genes that have undergone adaptive evolution may participate in the development of adaptive traits in P. euphratica and that PeuLAC2 could be a candidate gene for molecular genetic breeding in trees.
\end{abstract}

\section{Highlights}

- A LAC2 gene in P. euphratica underwent positive selection;

- PeULAC2-OE thickens the secondary cell wall;

- PeULAC2-OE improves drought tolerance in plants by enhancing their water transport capacity.

Correspondence: Dongshi Wan (wandsh@lzu.edu.cn)

'State Key Laboratory of Grassland Agro-Ecosystem, School of Life Sciences, Lanzhou University, Lanzhou, Gansu, People's Republic of China

${ }^{2}$ Laboratory of Cell Biology, College of Life Science and Technology, Huazhong

Agricultural University, Wuhan, People's Republic of China

\section{Introduction}

Water is one of the foremost limiting factors for normal plant survival. Plants, especially those distributed in arid and semiarid regions, have evolved various strategies to prevent water loss or adapt to growth in conditions with water deficiency ${ }^{1}$. For example, at the physiological level, root hydrotropic growth is stronger in desert poplar than in other poplar plants, and its exhibits high antioxidant enzyme activities ${ }^{2}$. At the morphological level, water is stored in the large parenchymal cells in the swollen stems

\section{(c) The Author(s) 2021}

(c) (i) Open Access This article is licensed under a Creative Commons Attribution 4.0 International License, which permits use, sharing, adaptation, distribution and reproduction cc) in any medium or format, as long as you give appropriate credit to the original author(s) and the source, provide a link to the Creative Commons license, and indicate if changes were made. The images or other third party material in this article are included in the article's Creative Commons license, unless indicated otherwise in a credit line to the material. If material is not included in the article's Creative Commons license and your intended use is not permitted by statutory regulation or exceeds the permitted use, you will need to obtain permission directly from the copyright holder. To view a copy of this license, visit http://creativecommons.org/licenses/by/4.0/. 
and leaves of succulent cacti. The closure or opening of stomata regulated by phytohormones ${ }^{3}$, together with the water transport capacity of stems (xylem pressure), promotes water balance in plants ${ }^{4}$. In woody plants, several studies have shown that tolerance to drought is strongly related to the structure of xylem, which is associated with the water transport capacity of plants ${ }^{5}$. However, experimental cases supporting the related evolutionary processes, especially those concerning the molecular regulatory mechanisms of desert poplar, remain limited.

LACCASEs $(L A C s)$ encode multicopper oxidases that can catalyze the oxidation of various substrates and reduce molecular oxygen $\left(\mathrm{O}_{2}\right)$ to water $\left(\mathrm{H}_{2} \mathrm{O}\right)^{6}$. LACs have been found widely in fungi, bacteria, insects, and plants $^{7,8}$. In plants, $L A C s$ are primarily involved in monomer polymerization to form phenolic biopolymers ${ }^{9,10}$. Increasing studies have found that LACs may participate in lignin synthesis and metabolism ${ }^{11,12}$. For example, in Arabidopsis, a total of 17 LAC members have been identified ${ }^{12}$, four of which (LAC4, LAC11, LAC15, and $L A C 17)$ are involved in lignin biosynthesis and regulating cell wall structure ${ }^{11,13,14}$. LAC1 from Pyrus bretschneideri has been interfamily-transferred into Arabidopsis, which induced a significant increase in the lignin content and cell wall thickness of interfascicular fibers and xylem cells ${ }^{15}$. In Populus trichocarpa, LAC3 is involved in the structural formation of normal cell walls and enhances the integrity of xylem fibers ${ }^{16}$. The expression of $L A C s$ can also be regulated by microRNAs; for example, Ptr-miR397a affects lignin content by negatively regulating $L A C$ genes in $P$. trichocarpa ${ }^{17}$, while miR397b modulates a $L A C$, which results in both increased lignin content and seed number in Arabidopsis $^{18}$. In rice, microRNA-directed LACCASE gene silencing alters lignification and contributes to the domestication of cultivated indica rice ${ }^{19}$. Lignification in plants is often associated with improved tolerance to biotic stresses. For example, the overexpression of GhLAC1 from Gossypium hirsutum enhances lignification and leads to increased tolerance to biotic stresses, such as cotton bollworms, cotton aphids, and fungal pathogens ${ }^{20}$. The overexpression of GhLAC15 enhances resistance to Verticillium wilt by increasing lignification and lignin content in plant cell walls ${ }^{21}$. Moreover, LACs also participate in the plant response to abiotic stresses ${ }^{12}$. In Arabidopsis, Atlac2 plants exhibit reduced root elongation under dehydration conditions, while Atlac 8 plants flower earlier than wild-type plants ${ }^{22}$. LACs may also be involved in plant growth through the regulation of the flavonoid pathway. For example, TRANSPARENT TESTA10, a LAClike enzyme, is related to the oxidative polymerization of flavonoids in the Arabidopsis seed coat ${ }^{9}$. Rice OsChI1, a putative $L A C$ precursor that is overexpressed, increases tolerance to salt and drought stresses in Arabidopsis ${ }^{23}$.
OsLAC10 can reduce $\mathrm{Cu}$ uptake into roots, is associated with lignin synthesis, and further contributes to the increased tolerance to $\mathrm{Cu}$ in Arabidopsis ${ }^{10}$.

$L A C s$ are involved in regulating a series of metabolic pathways in plant development and growth; but the functional redundancy among members of the $L A C$ family is pervasive ${ }^{12,14}$, which may result in functional divergence among the $L A C$ family members in plants. However, such divergence has not yet been clearly described. Here, we investigated the $L A C$ family in $P$. euphratica (desert poplar). This species grows mainly in western China and adjacent Middle-Eastern regions ${ }^{24}$ and exhibits strong tolerance to severe drought and salinity $^{25}$. P. euphratica provides multiple ecosystem services as a natural barrier to the expansion of deserts, such as resisting sandstorms, regulating oasis climates, and even maintaining the ecosystem balance ${ }^{24}$. Thus, $P$. euphratica is widely considered a model woody plant for the study of the abiotic resistance mechanisms of trees $^{26}$. P. euphratica has evolved many strategies to adapt to the severe desert environment ${ }^{25}$. Among them, the polymorphisms in its leaves ${ }^{27}$ and its hard wood are considered two important adaptive traits ${ }^{28}$ that may provide desert poplars with increased flexibility to adapt to desert environments ${ }^{29}$. For example, $P$. euphratica wood can accumulate substantial amounts of cellulose and lignin in its xylem. Within the xylem, the secondary cell wall (SCW) not only provides mechanical support for plants ${ }^{30}$ and acts as a defense barrier to pathogen and insect attacks $\mathrm{s}^{31,32}$ but also provides a channel for the long-distance transportation of nutrients and water. Altogether with the polymorphisms in leaves, these traits allow the plants to exhibit physiological responses that help them adapt to extremely arid environments, including decreased photosynthetic activity, stomatal aperture control, and altered cell wall elasticity ${ }^{33}$. However, the molecular adaptive mechanisms underlying these physiological responses remain unknown.

In this work, we identified members of the $L A C$ family in the $P$. euphratica genome and performed phylogenetic and evolutionary analyses. The $L A C 2$ gene, which has been subjected to positive selection, was chosen and used for genetic and functional analyses in $P$. alba var. pyramidalis and Arabidopsis thaliana (Col-0), respectively. Our study provides insights into the adaptive evolution of key genes that provide important genetic resources for the adaptation of desert poplar to extremely arid environments.

\section{Results}

Identification of $\angle A C$ genes from the $P$. euphratica genome

We identified the $L A C$ family through a BLASTp search using 17 Arabidopsis LAC protein sequences to BLAST against the $P$. euphratica genome. The Pfam and SMART 


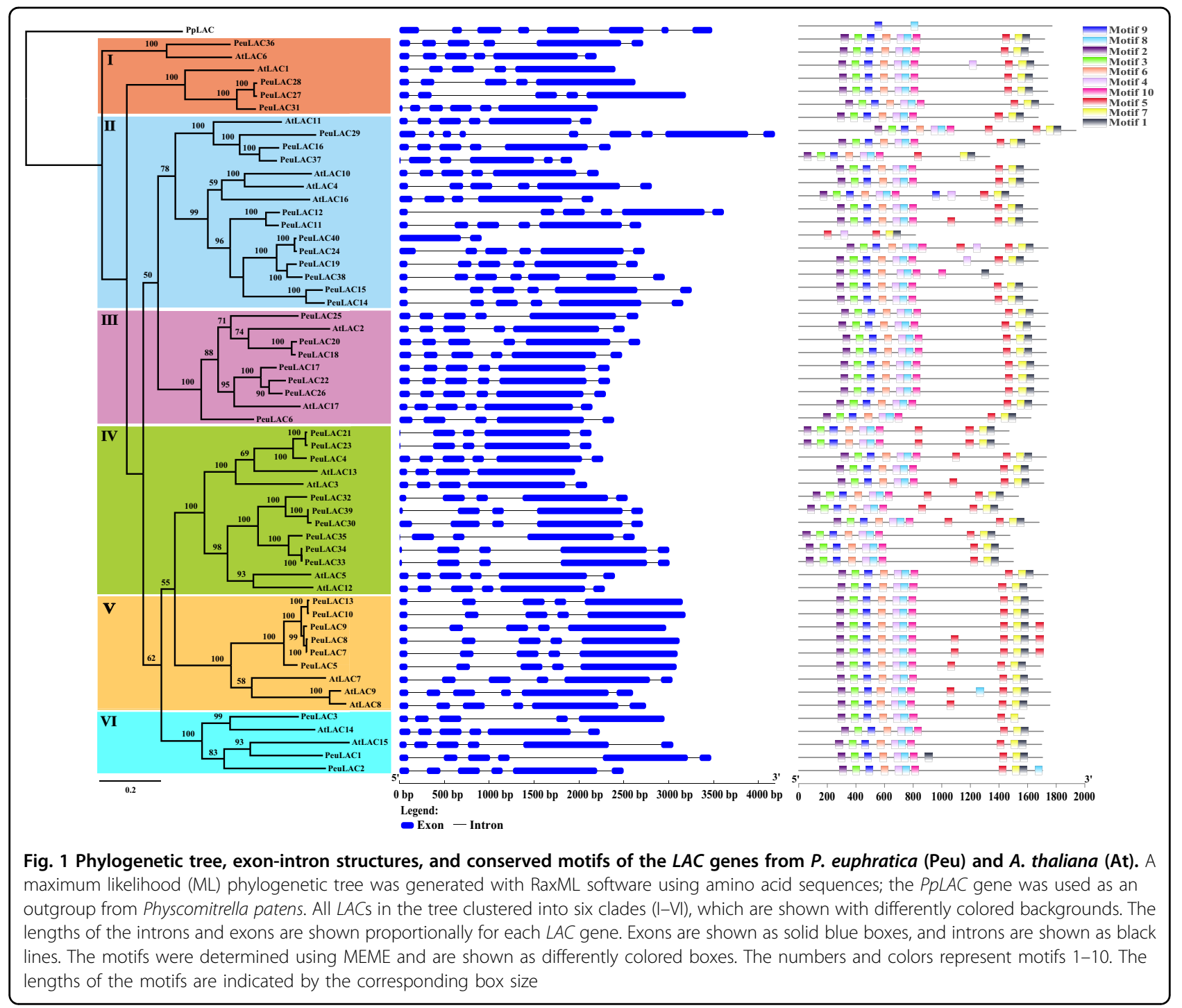

databases were used to search for conserved domains, and 40 LAC genes with $\mathrm{Cu}$-oxidase domains, named PeuLAC1-PeuLAC4O (Table S1 and Supplementary Data 1 ), were identified for phylogenetic analyses. An ML tree was generated using RaxML, with PpLAC from Physcomitrella patens as the outgroup (Fig. 1). In the ML tree, all $L A C$ s clustered into six clades (I-VI). In each clade, $L A C s$ from $P$. euphratica had more copies than those from Arabidopsis and were grouped into 13 gene pairs with high bootstrap values (Fig. 1).

The gene structures of PeuLACs were investigated using GSDS (Fig. 1). The PeuLACs showed varying intron-exon patterns, and the exon number ranged from 2 to 9 . A total of 10 conserved motifs in PeuLACs were detected using MEME (Fig. 1). The analyses of the biochemical characteristics showed that the molecular masses of PeuLACs ranged from $29.38 \mathrm{kDa}$ (PeuLAC40) to $71.44 \mathrm{kDa}$ (PeuLAC29), the predicted theoretical isoelectric points (pIs) ranged from 6.41 (PeuLAC2) to 9.82 (PeuLAC6), and most PeuLACs consisted of between 550 and 580 amino acid residues (Table S1). Signal peptides at the Nterminus were predicted in 25 PeuLACs. Furthermore, we predicted the phosphorylation sites and variable $N$ - or $O$-glycosylation sites in all PeuLAC proteins (except PeuLAC40), indicating the potential for posttranslational modification (Table S1).

The tissue-specific expression patterns of the PeuLAC gene family in P. euphratica were investigated from RNAseq data $^{34}$. The results showed that PeuLACs were expressed in various tissues (roots, leaves, xylem, and phloem) and were regulated by $\mathrm{NaCl}$ treatments (150 and $300 \mathrm{mM} \mathrm{NaCl}$, respectively). However, in clade I (except PeuLAC36) and clade V, four gene pairs, PeuLAC24/40, PeuLAC22/26, PeuLAC30/39, and PeuLAC33/34, had similar expression patterns, while PeuLAC16/37, Peu$L A C 19 / 38$, and PeuLAC1/2 showed significant differences 


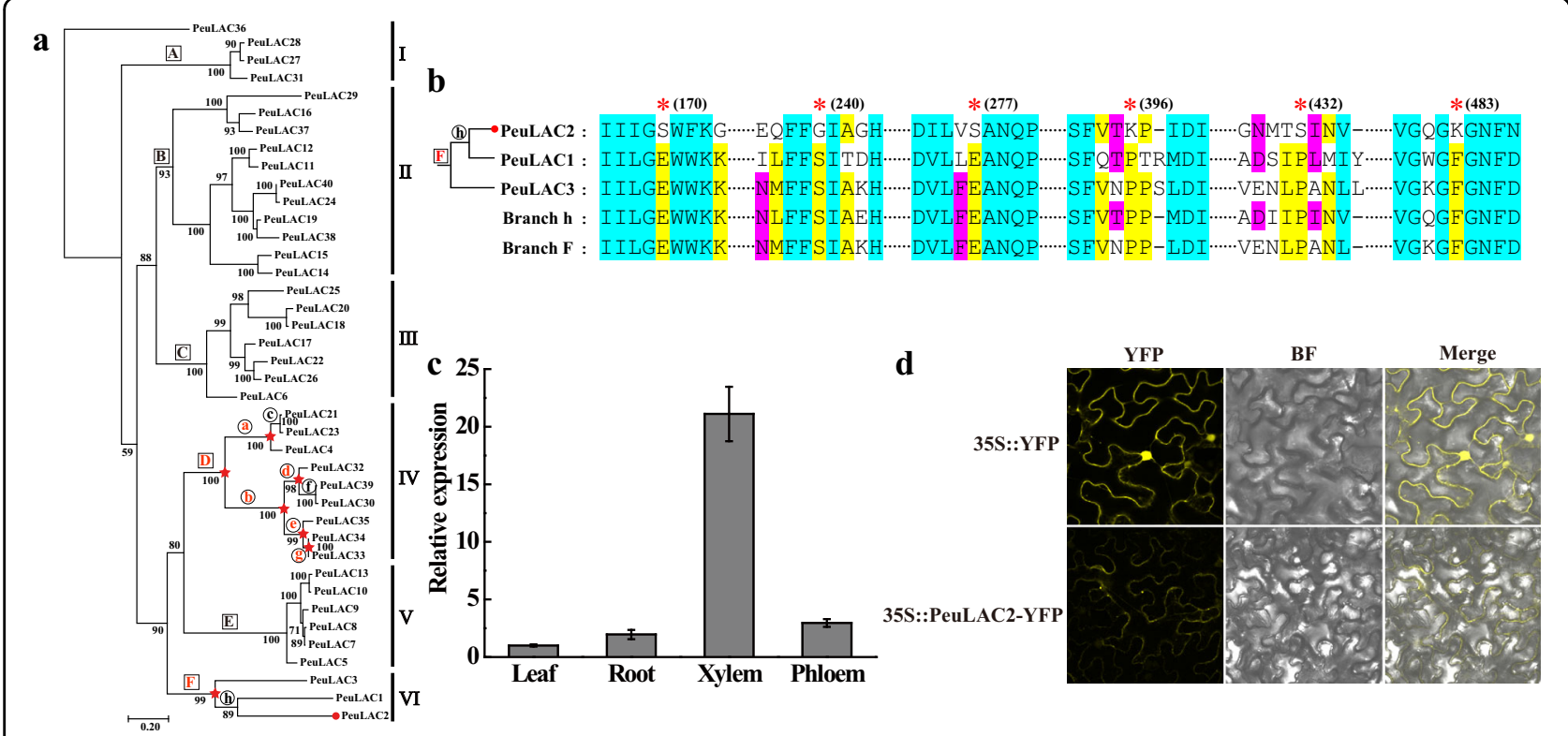

Fig. 2 Adaptive evolution analysis of the LAC family in P. euphratica, expression pattern and subcellular localization of PeuLAC2. a A maximum likelihood (ML) phylogenetic tree of all 40 PeuLACs. All PeuLAC genes clustered into six clades (I-VI). Red letters and red stars represent the signals of positive selection detected in ancestral branches (branches $D$ and $F$ and subbranches $a, b, d, e$, and g). In branches $A, B, C$, and E and subbranches $\mathrm{C}, \mathrm{f}$, and $\mathrm{h}$, no signals of positive selection were detected. The red circle represents the signal of positive selection detected in terminal branch PeuLAC2. b Sequence alignments and six positive selection sites. Alignments of PeuLACs in clade VI terminal branches (PeuLAC1, PeuLAC2, PeuLAC3) and ancestral branches (branch F and branch h) were generated by DNAMAN software. Positive selection sites are indicated with asterisks. c Expression of PeuLAC2 examined by qRT-PCR in the leaves, roots, xylem and phloem of P. euphratica. d Subcellular localization of the PeuLAC2 protein; $48 \mathrm{~h}$ after transient expression in Nicotiana benthamiana, confocal images of PeuLAC2 fused to an N-terminal YFP and of the control 35S::YFP were obtained. YFP-only images, brightfield images, and merged images are shown

(Fig. S1). These results suggest not only possible functional redundancy between the gene pairs but also functional divergence among the duplicates.

\section{Analysis of the adaptive evolution of the LAC family in P. euphratica}

To examine whether some PeuLACs experienced adaptive evolution, putative positive selection was investigated using a two-step branch-site model from CODEML in PAML ${ }^{35}$. Based on the ML tree (Fig. 2a), signals of positive selection were detected in branches $\mathrm{D}$ (clade IV, $P<0.001$ ) and F (clade VI, $P<0.001$ ) (Fig. 2a and Table 1). Further analysis using a BEB procedure revealed four sites (alignment positions 22, 65, 85, and 92) with posterior probabilities $\geq 0.99$ that apparently underwent positive selection in the ancestral branch of branch $F$ (clade VI) (Table 1). Then, we detected all terminal and ancestral branches in clade IV and clade VI, which exhibited positive selection in their ancestral nodes. In clade VI, we found a positive selection signal only in the terminal branch of PeuLAC2 $\left(P=0.013, \omega_{2}=88.382\right)$ and at six sites (alignment positions 170, 240, 277, 396, 432, and 483) with posterior probabilities $\geq 0.80$ (Fig. $2 \mathrm{~b}$ and Table 1 ). In clade IV, positive selection was also detected in the ancestral branch of other branches (branches a, b, d, e, and g)
(Fig. 2a and Table 1), but no positive selection signals were detected in the terminal branches of clade IV.

PeULAC2 is expressed ectopically in Arabidopsis and poplar

To investigate the putative function of PeuLAC2, full-length PeuLAC2 cDNA was cloned into the pBIBBASTA-35S-GWR-GFP vector by the Gateway method. The resulting construct vector was then transformed into Arabidopsis and P. alba and used to generate 18 PeuLAC2OE Arabidopsis lines and 16 PeuLAC2-OE P. alba lines, respectively. Under normal growth conditions, there were no significant changes in the phenotypes of transgenic poplar and Arabidopsis plants compared with those of WT plants. The expression level of PeuLAC2 in the transgenic lines was analyzed by quantitative reverse transcription PCR (qRT-PCR) (Fig. S5). Of these transgenic lines, the AtOE4, At-OE6, and At-OE9 lines of Arabidopsis and the PalOE2, Pal-OE5, and Pal-OE8 lines of P. alba, which exhibited high, moderate, and low expression levels, respectively (Fig. S5), were selected for subsequent analyses.

The expression of PeuLAC2 among tissues in $P$. euphratica was confirmed by qRT-PCR to occur mainly in the xylem (Fig. 2c). The subcellular localization of PeuLAC2 was examined in $N$. benthamiana epidermal cells. The fusion vector 35S::PeuLAC2-YFP and the control 
Table 1 Analysis of positive selection in PeuLAC family genes in P. euphratica using a branch-site PAML model

\begin{tabular}{|c|c|c|c|c|c|}
\hline Branch-site Model & $-\ln L$ & $2 \triangle(\ln L)$ & $P$-value & Parameter estimates & Positively selected sites \\
\hline \multicolumn{6}{|c|}{ Dataset I: all PeuLACs (40 sequences) } \\
\hline \multicolumn{6}{|l|}{ Branch F } \\
\hline Null & 6929.287611 & & & $\omega_{0}=0.11, \omega_{1}=1.0, \omega_{2}=1.0$ & \\
\hline Alternative & 6922.843499 & 12.89 & $<0.001$ & $\begin{array}{l}\omega_{0}=0.11, \omega_{1}=1.0 \\
\omega_{2}=999.000\end{array}$ & $\begin{array}{l}22 Y\left(0.990^{*}\right) ; \text { 65S }\left(0.999^{* *}\right) \\
85 \mathrm{~S}\left(0.993^{* *}\right) ; 92 \mathrm{D}\left(0.993^{* *}\right)\end{array}$ \\
\hline \multicolumn{6}{|l|}{ Branch D } \\
\hline Null & 6931.431556 & & & $\omega_{0}=0.11, \omega_{1}=1.0, \omega_{2}=1.0$ & \\
\hline Alternative & 6920.259594 & 22.34 & $<0.001$ & $\begin{array}{l}\omega_{0}=0.11, \omega_{1}=1.0 \\
\omega_{2}=999.000\end{array}$ & \\
\hline \multicolumn{6}{|c|}{ Dataset II: clade VI (3 sequences) } \\
\hline \multicolumn{6}{|c|}{ Branch (terminal branch of PeuLAC2) } \\
\hline Null & 4739.621196 & & & $\omega_{0}=0.06, \omega_{1}=1.0, \omega_{2}=1.0$ & \\
\hline Alternative & 4736.546196 & 6.15 & $=0.013$ & $\begin{array}{l}\omega_{0}=0.07, \omega_{1}=1.0, \\
\omega_{2}=88.382\end{array}$ & $\begin{array}{l}170 \mathrm{~S}\left(0.867^{*}\right) ; 240 \mathrm{G}\left(0.856^{*}\right) \\
277 \mathrm{~S}\left(0.824^{*}\right) ; 396 \mathrm{~K}\left(0.842^{*}\right) ; \\
432 \mathrm{~S}\left(0.832^{*}\right) ; 483 \mathrm{~K}\left(0.890^{*}\right)\end{array}$ \\
\hline \multicolumn{6}{|c|}{ Dataset III: clade IV (9 sequences) } \\
\hline \multicolumn{6}{|l|}{ Branch a } \\
\hline Null & 5541.400987 & & & $\omega_{0}=0.07, \omega_{1}=1.0, \omega_{2}=1.0$ & \\
\hline Alternative & 5534.424466 & 13.95 & $<0.001$ & $\begin{array}{l}\omega_{0}=0.07, \omega_{1}=1.0 \\
\omega_{2}=13.415\end{array}$ & \\
\hline \multicolumn{6}{|l|}{ Branch b } \\
\hline Null & 5537.882645 & & & $\omega_{0}=0.06, \omega_{1}=1.0, \omega_{2}=1.0$ & \\
\hline Alternative & 5532.622033 & 10.52 & $<0.001$ & $\omega_{0}=0.07, \omega_{1}=1.0, \omega_{2}=4.560$ & \\
\hline \multicolumn{6}{|l|}{ Branch e } \\
\hline Null & 5555.958312 & & & $\omega_{0}=0.07, \omega_{1}=1.0, \omega_{2}=1.0$ & \\
\hline Alternative & 5549.757667 & 12.40 & $<0.001$ & $\begin{array}{l}\omega_{0}=0.08, \omega_{1}=1.0 \\
\omega_{2}=998.990\end{array}$ & \\
\hline \multicolumn{6}{|l|}{ Branch d } \\
\hline Null & 5556.551602 & & & $\omega_{0}=0.08, \omega_{1}=1.0, \omega_{2}=1.0$ & \\
\hline Alternative & 5549.891034 & 13.32 & $<0.001$ & $\begin{array}{l}\omega_{0}=0.08, \omega_{1}=1.0 \\
\omega_{2}=998.997\end{array}$ & \\
\hline \multicolumn{6}{|l|}{ Branch $g$} \\
\hline Null & 5557.376628 & & & $\omega_{0}=0.08, \omega_{1}=1.0, \omega_{2}=1.0$ & \\
\hline Alternative & 5553.172610 & 8.41 & $<0.001$ & $\begin{array}{l}\omega_{0}=0.08, \omega_{1}=1.0, \\
\omega_{2}=119.419\end{array}$ & \\
\hline
\end{tabular}

Note: Codons identified by PAML as under positive selection along with Bayesian (BEB) analysis posterior probability for sites with $P>0.80$ under branch-site models $(*, 0.99>P>0.80 ; * *, P>0.99)$

35S::YFP were transiently expressed. Observation by confocal laser-scanning microscopy revealed that the yellow fluorescence of the 35S::PeuLAC2-YFP fusion protein was localized exclusively in the membrane of $N$. benthamiana epidermal cells, whereas the control YFP protein was localized in both the membrane and the nucleus (Fig. 2d).
Drought tolerance in Arabidopsis and poplar with PeuLAC2OE

Previous studies have shown that $L A C$ s participate in the plant response to abiotic stresses ${ }^{12}$. To investigate the role of PeuLAC2 in drought tolerance, we investigated the PeuLAC2 expression levels in the leaves, roots, xylem, and 
phloem of $P$. euphratica subjected to a drought treatment for 0 or 15 days. The results showed that the drought treatment significantly induced the expression of PeuLAC2 in sapling leaves and xylem, while no significant change was observed in the roots and phloem (Fig. S2). Furthermore, we also assessed the drought sensitivity of transgenic plants. PeuLAC2-OE Arabidopsis plants were stressed by withholding water for 18 days and then rehydrated for 2 days. The survival rate was determined according to the standard that surviving plants could grow new leaves and grow normally. After 18 days without being watered, PeuLAC2-OE and WT Arabidopsis plants showed symptoms of drought-induced damage such as leaf rolling and wilting, but PeuLAC2-OE plants showed only slight symptoms and At-WT showed visible symptoms (Fig. S3a). After rewatering for 2 days, the At-OE4, At-OE6 and AtOE9 plants survived at rates of $63.9 \%, 62.5 \%$, and $58.3 \%$, respectively, which were all higher than the survival rate of At-WT (22.2\%) (Fig. S3b). In terms of the water loss rate, the three At-OE plants maintained a reduced rate of water loss and showed higher drought tolerance than At-WT (Fig. S3c). Similar results were also found in terms of the leaf RWC (Fig. S3d). These results indicated that PeuLAC2 overexpression improved drought tolerance in Arabidopsis.

To investigate whether PeuLAC2 is involved in drought-induced oxidative stress, we examined the related parameters in PeuLAC2-OE and WT Arabidopsis plants treated by withholding water for 0,12 , and 15 days. The results showed that the MDA concentration was significantly higher in At-WT than in At-OE plants after 12 days without watering (Fig. S3e). The PRO level was lower in At-WT than in At-OE plants after being treated for 12 and 15 days (Fig. S3f). The $\mathrm{H}_{2} \mathrm{O}_{2}$ content was higher in At-WT than in At-OE plants after 15 days without watering, but this difference was not significant (Fig. S3g). The activities of POD and SOD, which contribute to avoiding oxidative damage induced by drought stress, increased significantly in the At-OE plants (Fig. S3h, S3i). These results indicated that PeuLAC2 overexpression increased antioxidant enzyme activities and decreased oxidative damage under drought stress.

We then analyzed drought tolerance in poplar that exhibited PeuLAC2 overexpression. When the Pal-OE and Pal-WT plants were stressed for 10 days without watering, the Pal-WT plants showed severe symptoms of drought-induced damage, such as leaf rolling and wilting, but Pal-OE poplars wilted to a much lesser extent than Pal-WT poplars (Fig. 3a). After 16 days without watering followed by rewatering for 5 days, the survival rate of the Pal-OE plants was significantly higher than that of the Pal-WT plants (Fig. 3b). We also investigated drought-induced oxidative stress in poplars. When the Pal-OE and Pal-WT plants were treated for 10 days without watering, the Pal-OE plants displayed significantly lower MDA and $\mathrm{H}_{2} \mathrm{O}_{2}$ concentrations than the Pal-WT plants (Fig. 3c, d). The activity of CAT, which is the most important hydrogen peroxide-scavenging enzyme, was significantly higher in Pal-OE plants than in Pal-WT plants after 10 days without watering (Fig. 3e). These results indicated that PeuLAC2 overexpression improved plant drought tolerance by reducing oxidative damage under drought stress.

Previous studies have shown that higher stem xylem water potential can prevent hydraulic failure caused by drought and enhance drought tolerance ${ }^{36}$. We thus determined the effect of PeuLAC2 overexpression on physiological changes in poplar, which may contribute to drought survival. The stem xylem water potential of PeuLAC2-OE poplars was higher than that of Pal-WT poplars under drought stress (Fig. 3f). We examined the morphology of poplar stem xylem cells in Pal-OE and Pal-WT plants. In P. alba, the stem xylem vessels in the Pal-OE plants were smaller than those in the Pal-WT plants (Fig. 5a, b), while the Pal-OE plants had a much higher vessel number per unit of area than the Pal-WT plants, especially Pal-OE2 (Fig. 5a, c). The area of vessels in the transverse section of the woody stem was higher in the Pal-OE plants than in the Pal-WT plants (Fig. 5d). However, in Arabidopsis, the stem xylem vessels, the vessel number per unit of area, and the area of vessels in the transverse section in the At-OE plants were not significantly different from those in the At-WT plants (Figs. 4a and Fig. S4). These responses may contribute to improving the water transport capacity of the plants.

\section{Determination of SCW thickness and composition of xylem cells and stem tensile strength in Arabidopsis}

Previous studies have revealed that PbLAC1 overexpression in Arabidopsis significantly increases the cell wall thickness in the xylem fibers and interfascicular fibers ${ }^{15}$. In this study, we generated paraffin sections to observe the thickness of the cell wall in both the interfascicular and xylem fibers of Arabidopsis. Toluidine blue staining in Arabidopsis revealed that the SCW thickening of the fiber cells in At-WT plants was weaker than that in At-OE (Fig. 4a). These results were further verified by TEM in Arabidopsis (Fig. 4b, c). The vessel cell thickness did not differ significantly between At-OE and At-WT plants (Fig. 4b, c).

PeuLAC2 overexpression increased the SCW thickness in Arabidopsis regardless of whether it altered the cell wall composition. The examination of the cell wall composition revealed that in the At-OE4, At-OE6, and At-OE9 Arabidopsis plants, the lignin contents were 138.47, 153.19 , and $151.71 \mu \mathrm{g} / \mathrm{mg}$, respectively, which were significantly lower than those in At-WT $(187.23 \mu \mathrm{g} / \mathrm{mg})$ (Fig. 4d); the cellulose contents of the SCWs of these 


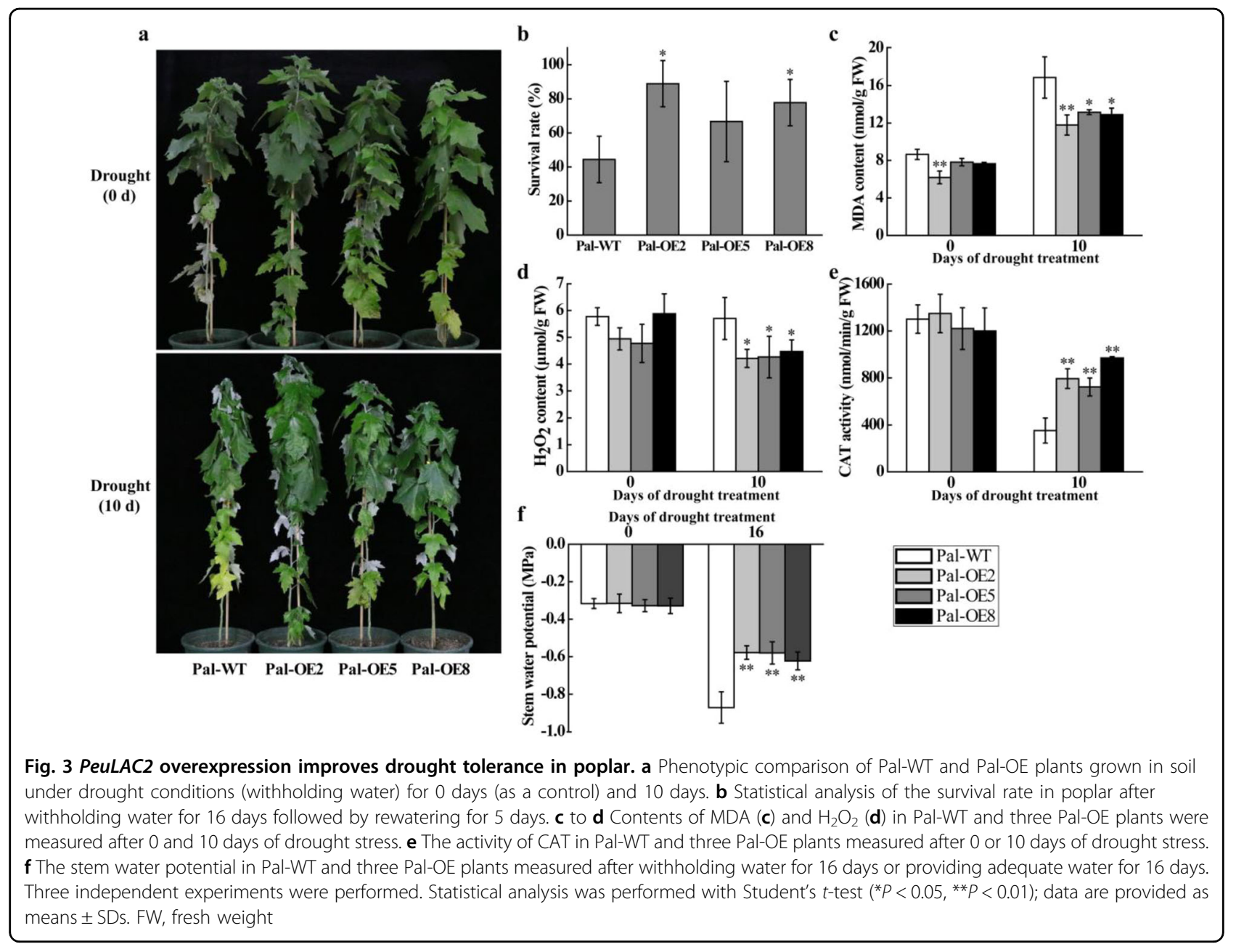

transgenic lines were $37.79,35.80$, and $32.87 \mathrm{mg} / \mathrm{g}$, respectively, which were significantly higher than that in At-WT $(32.19 \mathrm{mg} / \mathrm{g})$ (Fig. 4d). Moreover, the content of xylose, an important component of hemicellulose, increased significantly in transgenic plants (34.36, 33.32, and $34.69 \mu \mathrm{g} / \mathrm{g}$ for At-OE4, At-OE6, and At-OE9, respectively) compared with that in At-WT plants $(30.64 \mu \mathrm{g} / \mathrm{g})$ (Fig. 4e), while the contents of other hemicelluloses, such as arabinose, mannose, galactose, and glucose, showed no significant differences (Fig. 4e).

The overexpression of PeuLAC2 altered the cell wall composition and increased the cellulose and xylose contents of the interfascicular fibers and xylem cells in Arabidopsis. These fibers and cells are the main stem tissues supporting the upright growth of inflorescences. The tensile strength of the Arabidopsis inflorescence stem was measured, and the results indicated that the mechanical strength was higher in At-OE plants than in At-WT plants. The stem tensile strength of At-WT was defined as $100 \%$, and in the three transgenic lines (At-OE4, At-OE6, and At-OE9), the stem tensile strengths were calculated as $149.72 \%, 112.35 \%$, and $104.61 \%$, respectively (Fig. 4f). Furthermore, the inflorescence stem structure was examined, and the results showed that the fiber cells in the transgenic plants were longer (average lengths of 0.98 , 0.94, and $0.90 \mathrm{~mm}$ for At-OE4, At-OE6, and At-OE9, respectively) than those in the At-WT plants $(0.89 \mathrm{~mm})$ (Fig. 4g, h).

\section{Determination of SCW thickness and composition of xylem cells in poplar}

PeuLAC2 overexpression increased the SCW thickness and altered the cell wall composition of xylem cells in Arabidopsis; similar results were observed in poplar. We determined the SCW thickness and cell wall composition of xylem cells in the Pal-OE and Pal-WT poplars. The results indicated that the thickening of the SCWs of the fiber cells in the Pal-OE poplars was significantly greater than that in the Pal-WT poplars (Fig. 5a, e). Peu$L A C 2$ overexpression also reduced the vessel area and increased the vessel number per unit of area and the area of the vessels in the transverse section in poplar plants 


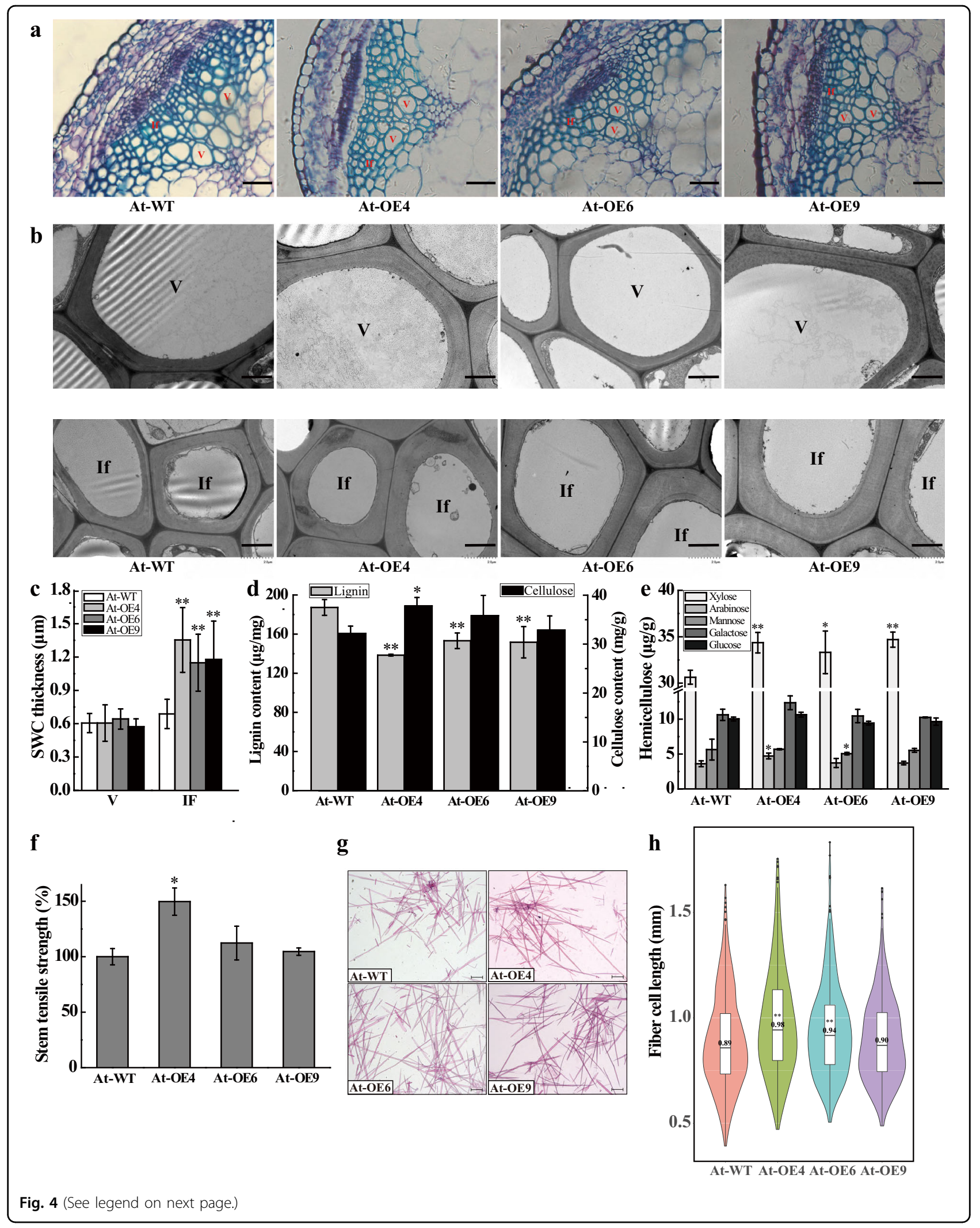


(see figure on previous page)

Fig. 4 PeuLAC2 overexpression enhances SCW thickness in fibers and affects SCW components in the inflorescence stem of Arabidopsis. a Cross-sections of Arabidopsis inflorescence stems in At-WT and three At-OE plants stained with $0.1 \%$ toluidine blue. V, vessel; If, interfascicular fiber cell. Bar $=20 \mu \mathrm{m}$. b Transmission electron micrographs (TEM) of the cell cross-sections shown in $\mathbf{a}$. V, vessel; If, interfascicular fiber cell. Bar $=2 \mu \mathrm{m}$. c The SCW thickness was measured in the vessel cells and interfascicular fiber cells in $\mathbf{b}$ by ImageJ software, and at least 30 cells in each of three plants were measured. $\mathbf{d}$ to e SCW components analyzed in the Arabidopsis inflorescence stem. Contents of lignin, cellulose (d) and hemicellulose (e). f Tensile strength analysis of the Arabidopsis inflorescence stem. More than 20 plants in each line were measured. $\mathbf{g}$ Fiber cells in Arabidopsis inflorescence stems were disaggregated and stained with safranin T. Bar $=1 \mathrm{~mm}$. $\mathbf{h}$ Statistical analysis of the fiber cell length in $\mathbf{g}$; at least 500 fiber cells were measured in each plant genotype. Three independent experiments were performed. Statistical analysis was performed with Student's $t$-test $\left({ }^{*} P<0.05,{ }^{*} P<0.01\right)$; data are provided as means \pm SDs

(Fig. 5b-d). The determination of the cell wall composition showed that PeuLAC2 overexpression also decreased the lignin content and increased the cellulose content in P. alba (Fig. 5f, g). However, with regard to hemicellulose, the xylose content increased in Pal-OE compared with that in Pal-WT, while the glucose, arabinose, mannose, and galactose contents in the Pal-OE plants showed no significant differences from those in the Pal-WT plants (Fig. 5h). These results showed that PeuLAC2 was involved in regulating $\mathrm{SCW}$ biosynthesis in interfascicular fiber and xylem cells.

We also determined the composition of the SCWs in poplar plants under drought conditions. For the drought treatment, two-month-old Pal-OE and Pal-WT poplar saplings were subjected to drought for 0 and 12 days, respectively, and the SCW composition of those sapling stems was examined. The results showed that PeuLAC2 overexpression significantly decreased the lignin content and increased the cellulose content of the SCWs in P. alba under drought for 0 and 12 days, respectively (Fig. 5f, g). However, for hemicellulose, PeuLAC2 overexpression significantly increased the xylose contents of SCWs under drought treatment (Fig. 5h); the contents of galactose, mannose, glucose, and arabinose in both Pal-OE and PalWT plants increased under drought conditions, but the differences in the increases between genotypes were not significant $(P>0.05)$ (Fig. 5h). These results further indicated that PeuLAC2-OE affects the contents of lignin, cellulose, and hemicellulose in SCWs, which may affect SCW functioning and might be involved in drought tolerance.

\section{RNA-seq analysis of PeuLAC2-OE plants to evaluate drought tolerance}

To further reveal the potential regulatory network through which PeuLAC2 is involved in drought tolerance, we conducted an RNA-seq analysis of PeuLAC2-OE Arabidopsis plants. After drought stress for 12 days, only 15 DEGs (adjusted $P$-value $<0.05)$ were identified, with seven upregulated genes and eight downregulated genes (Table S3). In the drought-treated At-OE plants, a total of 23 DEGs were detected (adjusted $P$-value $<0.1$ ), with 11 upregulated genes and 12 downregulated genes (Fig. 6a and Table S3). Among the upregulated genes, NAC025 (AT1G61110), UGT
(AT3G46700), and BG1 (AT3G57270) are associated with stress resistance and the synthesis of the cell wall components cellulose and xylose, which influence the cell wall structure. Among the downregulated genes, AT1G04470, a member of the DUF810 family, is related to stomatal responses as well as salt and drought tolerance (Table S3). qRT-PCR analysis further confirmed the expression levels of these genes in PeuLAC2-OE Arabidopsis plants. The results indicated that the expression of NACO25, BG1, and UGT was activated and that the expression of DUF810 (AT1G04470) was suppressed in transgenic Arabidopsis lines (Fig. 6b). We were interested in determining whether these up- and downregulated genes exhibited similar expression patterns in poplar plants under drought stress. Based on the NAC025, BG1, UGT, and DUF810 genes in Arabidopsis, we identified the corresponding orthologous genes in poplar: PAYT001587.1 (NAC025), PAYT006377.1 (BG1), PAYT023019.1 (UGT), and PAYT036935.1 (DUF810). qRT-PCR results revealed that the expression of PAYT001587.1 (NAC025), PAYT006377.1 (BG1), and PAYT023019.1 (UGT) was activated but that the expression of PAYT036935.1 (DUF810) did not change significantly in poplar plants under drought conditions (Fig. 6c).

\section{Discussion}

Drought can seriously limit the growth and development of plants. The laccase protein family is considered to be important in plant responses to various stres$\operatorname{ses}^{10,12,20,21,23}$. Previous evidence, including spatialtemporal expression patterns and expression regulation models, has shown that the functional redundancy among members of the $L A C$ family is pervasive in Arabidopsis $^{12,14}$. The functional redundancy of duplicated genes is widespread and provides an important source of genetic novelties ${ }^{37,38}$ upon which natural selection may act to contribute to the maintenance of partial gene redundancy ${ }^{38}$. Increasing amounts of experimental data indicate that redundant pairs are often associated with proteins involved in signaling and posttranslational protein modifications, such as phosphorylation and ubiquitination $^{39}$. However, less experimental evidence that could elucidate the roles of natural selection in redundant gene evolution is available. 


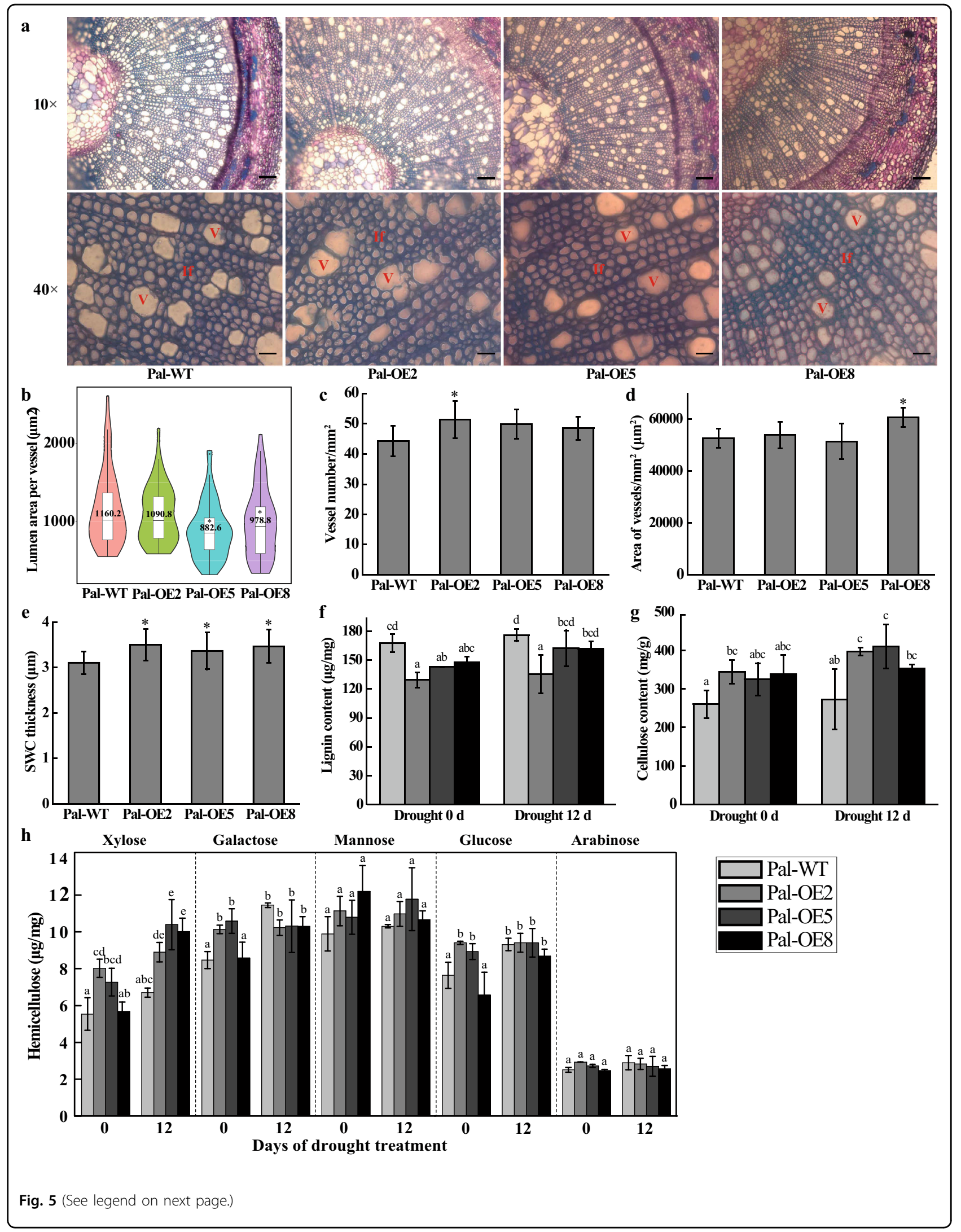


(see figure on previous page)

Fig. 5 PeuLAC2 overexpression enhances SCW thickness in fibers and affects the number and size of vessels in the xylem of $P$. alba. a Cross-sections of the fifth internode of the stem in Pal-WT and three Pal-OE P. alba plants stained with $0.1 \%$ toluidine blue and imaged at $\times 10$ magnification, Bar $=100 \mu \mathrm{m}$ and $\times 40$ magnification, Bar $=20 \mu \mathrm{m}$. b-d Mean lumen area of individual vessels $\left(\mu \mathrm{m}^{2}\right)(\mathbf{b})$, number of vessels per cross-sectional area $\left(\mathrm{mm}^{2}\right)(\mathbf{c})$, and area of vessels $\left(\mu \mathrm{m}^{2}\right)$ per cross-sectional area $\left(\mathrm{mm}^{2}\right)(\mathbf{d})$ for the vessel cells from $(\mathbf{a})$. More than 100 vessel cells in each of the three plants were measured. e SCW thickness of the xylem cells in $\mathbf{a}$; at least 30 cells in each of three plants were measured. $\mathbf{f}$ to $\mathbf{h}$ SCW components in the P. alba stem analyzed under normal and drought conditions. Contents of lignin (f), cellulose ( $\mathbf{g})$, and hemicellulose (h). Three independent experiments were performed. Statistical analysis was performed with Student's $t$-test $\left({ }^{*} P<0.05,{ }^{* *} P<0.01\right)$; data are provided as means \pm SDs

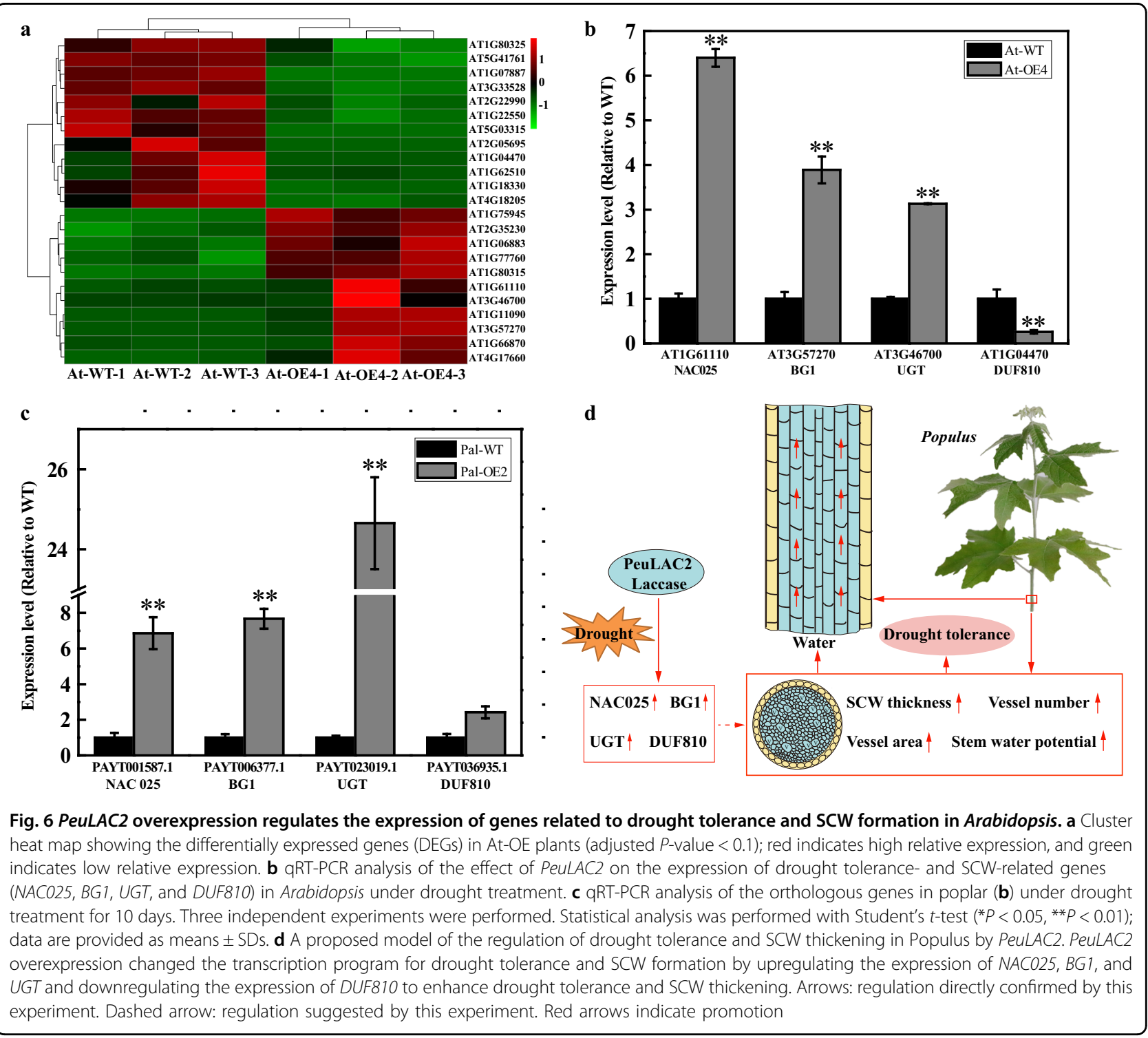

\section{Natural selection may contribute to the retention of $L A C$ gene redundancy}

LACs in P. euphratica have experienced multiple gene duplications, most of which likely occurred specifically in $P$. euphratica. Forty LACs from P. euphratica that clustered into six clades were identified based on amino acid sequence alignment with LAC-like multicopper oxidases, and most clades exhibited wider expansion than was observed in Arabidopsis LACs. LACs in P. euphratica, e.g., the gene pairs PeuLAC24 and PeuLAC40, PeuLAC19, and PeuLAC15 in clade II and the gene pairs PeuLAC30 and PeuLAC39, PeuLAC33, and PeuLAC34 in clade IV, had 
diverse sequences but exhibited highly conserved expression patterns after salt treatment (Fig. S1), showing apparent functional redundancy across a wide variety of tissues. Functional redundancies were also found among clades; for example, AtLAC4, AtLAC11, and AtLAC17 in clade III and AtLAC15 in clade VI have been shown to be related to lignin synthesis ${ }^{11,13,14}$. Evolutionary analysis showed that positive selection signals were detected in clade IV $(P<0.001)$ and clade VI $(P<0.001)$ (Fig. 2a and Table 1). In particular, in clade IV, both gene pairs exhibited redundant functions, indicating that natural selection might be involved in the retention of gene redundancy. In clade VI, three PeuLACs experienced different selective pressures and were expressed in different patterns. PeuLAC2 was subjected to positive selection, and six amino acid residues experienced adaptive evolution. The orthologous gene AtLAC14 in Arabidopsis is considered to be associated with stress responses $^{12}$, while $A t L A C 15$ is related to lignin synthesis and cell wall structure ${ }^{13}$; these results indicate that $L A C s$ in clade VI have diversified significantly in function. In addition, among all PeuLAC proteins (except PeuLAC40), phosphorylation sites and variable $N$ - or $O$-glycosylation sites have been found, indicating potential posttranslational modifications ${ }^{17}$. It is obvious that the expanded $L A C$ s in $P$. euphratica have experienced functional redundancy that would be associated with posttranslational protein phosphorylation modifications ${ }^{39}$.

\section{PeuLAC2 improves drought tolerance by enhancing water transport capacity}

As a multigene family, $L A C s$ have been verified to be associated with lignin polymerization ${ }^{11,14,17}$ and with responses to various stresses in plants ${ }^{10,12,20,21,23}$. Here, we found that PeuLAC2-OE lines had reduced water loss rates during drought stress (Fig. S3c). Consistent with the water loss rate, the leaf RWC was higher in the PeuLAC2OE plants than in the At-WT lines after drought treatment (Fig. S3d). These results indicated that PeuLAC2 overexpression reduced water loss by increasing RWC in Arabidopsis. In addition, the activities of POD and CAT were both enhanced in transgenic lines (Fig. S3f and Fig. 3e), resulting in reduced $\mathrm{H}_{2} \mathrm{O}_{2}$ accumulation (Fig. 3d) and indicating an enhanced antioxidant defense system in transgenic plants. The accumulation of high levels of reactive oxygen species (ROS) induced by drought stress not only damages cell membranes but also results in cell death $^{40}$. The overexpression of PeuLAC2 enhances antioxidant defense system activity to effectively protect membrane integrity and avoid damage to plants.

$L A C s$ are related to the formation of cell wall structures and lignin metabolism ${ }^{10,11,15,16}$. In this study, the Peu$L A C 2-\mathrm{OE}$ plants showed significantly increased SCW thickness in xylem cells in PeuLAC2-OE P. alba (Fig. 5e) and Arabidopsis (Fig. 4b, c). This result suggests that PeuLAC2 participates in the development of the SCW in xylem cells. In terms of the SCW components, the cellulose content was significantly higher in PeuLAC2-OE plants than in WT plants (Figs. 4d and 5g). Similar results were also found for another SCW component, hemicellulose. For example, xylose is one of the most abundant components of hemicellulose, and the xylose content was significantly increased in PeuLAC2-OE Arabidopsis plants (Fig. 4e). The contents of other hemicellulose components, including arabinose, mannose, galactose, and glucose, were not significantly different between genotypes (Fig. 4e). These results revealed that PeuLAC2 enhanced the cellulose and xylose contents and further increased SCW thickness in Arabidopsis. Both cellulose and xylose participate in cell wall formation. Celluloses, as the main component of the cell wall, have great mechanical strength and lead to high stem tensile strength in plants by increasing the length of fiber cells in the inflorescence stem. In addition, the hemicellulose and cellulose in plant cell walls are primarily consumed by the fungi that cause brown rot decay ${ }^{41}$; thus, the overexpression of PeuLAC2 might significantly improve plant resistance to fungi.

Furthermore, in plant conducting cells, the size and structure of stem xylem vessels can affect the water transport capacity of plants ${ }^{42}$. Hydraulic conductivity in xylem is associated with the water potential and vessel diameter $^{36,42}$. Under drought conditions, plants can regulate their hydraulic conductance as an adaptation to enhance drought tolerance ${ }^{4,43}$. The upward water transport through the xylem fails when a decrease in water potential reduces hydraulic conductivity; this phenomenon is called xylem cavitation or embolism ${ }^{36}$. Vessels with smaller diameters can endure lower water potentials to prevent xylem cavitation ${ }^{42}$. In PeuLAC2-OE P. alba plants under drought stress, the stem xylem water potential was higher (Fig. 3f), the vessel lumen area was smaller, and the number of vessel cells and area of vessels were much greater (Fig. $5 \mathrm{a}-\mathrm{d}$ ) than those in the Pal-WT controls; these differences contributed to enhancing the water transport capacity and led to improved drought tolerance in the transgenic poplars.

Increasing evidence indicates that laccase enzymes exhibit dual functions to catalyze both lignin biosynthesis and degradation ${ }^{44}$. These processes can be affected by multiple limiting factors, such as the $\mathrm{pH}^{45}$, threedimensional structure ${ }^{46}$, and C-terminus features ${ }^{46,47}$, which can all be changed by PeuLAC2 overexpression in plants. For example, among all of the LACs from $P$. euphratica, PeuLAC2 had the lowest theoretical isoelectric point (6.41) (Table S1), an additional motif 8 at the C-terminus (Fig. 1), and six adaptive amino acid sites (Fig. $2 \mathrm{~b}$ and Table 1). These features together might contribute to altering its function to negatively regulate 
lignin content, improve the water transport capacity and further enhance drought tolerance in plants.

\section{PeuLAC2 induces the expression of many genes involved in water transport in conducting cells}

A comparative transcriptome analysis of At-OE plants under drought conditions provided insight into the molecular mechanisms involved in the PeuLAC2-mediated drought-stress response and changes in SCW thickness. We identified DEGs from At-OE lines, and qRT-PCR analysis further confirmed the differential expression of AT1G04470, NAC025, $\beta$-1,3-GLUCANASE1 (BG1), and UGT (Fig. 6b). AT1G04470, a member of the DUF810 family, was suppressed in the At-OE plants under drought stress (Fig. $6 \mathrm{~b}$ and Table S3). In Arabidopsis, the DUF810 family gene and the PATROL1 mutation (patrol1) impaired the stomatal opening response $^{48}$. The rice gene OsDUF810.7 is involved in tolerance to salt and drought stresses $^{49}$. UDPglycosyltransferases (UGTs) glycosylate a wide range of metabolites and phytohormones in response to biotic and abiotic stresses. UGT74E2 overexpression increases the tolerance of Arabidopsis to drought and salinity stresses and reduces water loss in plants, while 11 UGTs are upregulated in response to hydrogen peroxide stress in plants deficient in catalase ${ }^{50}$. UGTS and $\beta-1,3-$ GLUCA$N A S E 1$ are also involved in cellulose and xylose synthesis, which influences the SCW structure ${ }^{51}$. NAC proteins play diverse roles in plant growth, development, and defense ${ }^{52}$, e.g., NAC, VND6, VND7, NST1, and SND1 are involved in SCW synthesis ${ }^{53}$. Based on the four genes in Arabidopsis, we identified the corresponding orthologous genes in poplar. The expression of the four genes in transgenic Arabidopsis and poplar was verified using qRT-PCR (Fig. 6b, c). NAC025, BG1, and UGT were upregulated in both Arabidopsis and poplar plants, showing similar expression patterns. However, DUF810 was downregulated in Arabidopsis, but there was no significant change in its expression in poplar. One possible explanation for this difference is that DUF810 shows different expression patterns in herbaceous and woody plants under drought stress. Therefore, we propose a simple model of how PeuLAC2 regulates drought tolerance by improving the water transport capacity in poplar (Fig. 6d). In the model, PeuLAC2 overexpression changes the transcription of several genes associated with SCW structure formation and stress responses, such as NAC025, BG1, UGT, and DUF810, to enhance drought tolerance.

Taken together, from an evolutionary perspective, redundancies due to gene duplication likely increased the ability of desert poplar (P. euphratica) to evolve multiple adaptive traits. For example, $P$. euphratica wood is rigid and dense, and substantial amounts of lignin and cellulose accumulate in the xylem; together with the SCW, this accumulation not only provides mechanical support for plants $^{30}$ and a defense barrier ${ }^{31,41}$ but also prevents excessive water evaporation from the plant, thus increasing the likelihood of the plant's survival in desert ecosystems $^{25,32}$. Collectively, our results provide evidence that PeuLAC2, underwent adaptive evolution, contributes to the thickening of SCWs by enhancing their cellulose and xylose contents, which may enhance the water transport capacity of $P$. euphratica and improve its tolerance to drought stress. Our data also contribute to clarifying the genetic mechanisms underlying drought tolerance in P. euphratica and suggest that PeuLAC2 could be an attractive candidate gene for molecular breeding programs for trees.

\section{Methods and materials}

\section{Identification of $L A C s$ in $P$. euphratica}

$P$. euphratica genome databases were used to collect amino acid sequences of $L A C$ (PeuLAC) genes. A BLASTp search was performed in the whole-genome database using all 17 amino acid sequences of Arabidopsis LACs as queries $^{12}$. SMART software (http://smart.embl-heidelberg.de/) and the Pfam database (http://pfam.xfam.org) were used to validate potential LACs identified in the $P$. euphratica genome. Protein queries that did not contain the known conserved domains were removed. A maximum likelihood (ML) phylogenetic tree was generated using amino acid sequences with RaxML software. Through the comparison of each putative PeuLAC coding sequence with its genomic sequence by the Gene Structure Display Server (GSDS v2.0; http://gsds.cbi.pku.edu.cn/), we identified the PeuLAC gene structures. The possible conserved motifs of the PeuLAC genes were predicted using MEME server v4.11.4 with the default parameters (http://meme-suite.org/tools/meme), except for the maximum number of identified motifs, which was set as 10 .

The physical and chemical characteristics of the PeuLACs were analyzed using ProtParamtool (http://web.expasy.org/ protparam). Potential glycosylation sites were predicted using the YinOYang 1.2 server (http://www.cbs.dtu.dk/ services/YinOYang/) and the NetNGlyc 1.0 server (http:// www.cbs.dtu.dk/services/NetNGlyc/). Putative signal peptide cleavage sites were analyzed using the SignalP 5.0 server (http://www.cbs.dtu.dk/services/SignalP/). Phosphorylation sites were analyzed using the NetPhos 3.1 server (http:// www.cbs.dtu.dk/services/NetPhos/).

\section{Molecular evolutionary analysis}

Codon-based ML models were used to estimate the rates of nonsynonymous substitutions $\left(d_{\mathrm{N}}\right)$ and synonymous substitutions $\left(d_{\mathrm{S}}\right)$ and the $d_{\mathrm{N}} / d_{\mathrm{S}}$ ratio (omega, $\left.\omega\right)$ in the CODEML program in PAML $4.7^{35}$. The ratio of nonsynonymous to synonymous substitutions $(\omega)$ was used to estimate the change in selective pressure, where 
$\omega=1, \omega<1$, and $\omega>1$ correspond to neutral, purifying, and positive selection, respectively. The PeuLAC ML tree was used as an input tree in all analyses (Fig. 2a). Branchsite model A was used to estimate positive selection, in which the model $\omega$ could vary among sites along specific lineages. Positive selection analysis for every gene in each foreground lineage was performed using the modified branch-site model A. The likelihood ratio test (LRT) statistic $(2 \Delta L)$ approximating a chi-square distribution was used to compare nested likelihood models. Bayes empirical Bayes (BEB) analysis ${ }^{35}$ was used to identify all positively selected sites in the branch-site models with posterior probabilities $\geq 0.80$.

\section{Plant materials}

Arabidopsis seeds of WT (Col-0, At-WT) and three transgenic lines of PeuLAC2-overexpression (At-OE) were surface-sterilized using $70 \%(\mathrm{v} / \mathrm{v})$ ethanol for $45 \mathrm{~s}$ and then with $1 \%$ sodium hypochlorite for $10 \mathrm{~min}$; finally, they were washed five times in sterilized water. The surface-sterilized seeds were vernalized at $4{ }^{\circ} \mathrm{C}$ for 2 days in the dark to break dormancy and were grown on MS medium containing $1 \%(\mathrm{w} / \mathrm{v})$ sucrose and $0.8 \%(\mathrm{w} / \mathrm{v})$ agar (pH 5.8) in a growth chamber at $22^{\circ} \mathrm{C}$ and $60 \%$ relative humidity (RH) under a 16/8 h (light/dark) cycle with 4500 lux light. For poplars, PeuLAC2 transgenic overexpression lines (Pal-OE) and WT (Pal-WT) P. alba saplings were propagated by in vitro microcutting. For the clonal propagation of sterilized transgenic and WT P. alba saplings, shoot segments of $2-3 \mathrm{~cm}$ were cut and cultivated on MS medium in a growth chamber at $23-25{ }^{\circ} \mathrm{C}$ and $60 \%$ relative humidity (RH) under a $14 / 10 \mathrm{~h}$ (light/dark) cycle under 4500 lux light.

\section{Plasmid construction and plant transformation}

Total RNA was extracted from P. euphratica leaves and used for reverse transcription analysis. To generate the OE plants, the full-length 1704-bp coding sequence of PeuLAC2 was amplified with specific primers (Table S2) and then cloned into the donor vector $\mathrm{pDONR/Zeo} \mathrm{via}$ the Gateway method (Invitrogen, Germany). The obtained entry clones were used to transfer the target sequence into the destination vector. In this study, the binary destination vector $p B I B-B A S T A-35 S-G F P$ with the CaMV $35 S$ promoter and BASTA herbicide resistance was used to express 35S::PeuLAC2 in plants. Agrobacterium tumefaciens strain GV3101 with the construct 35S::PeuLAC2 was used to transform WT Arabidopsis by floral dip transformation ${ }^{54}$. The transformed plants were identified by T2 progeny that were resistant to BASTA, and T3 transgenic plants were confirmed using PCR. For poplar, Agrobacterium-mediated infiltration of leaf discs was used to generate stably transgenic $P$. alba plants $^{55,56}$. Positive transgenic poplar plants were identified using BASTA herbicide, and PCR genotyping with specific primers for the PeuLAC2 gene (Table S2) was performed to identify the positive transgenic plants.

\section{Subcellular localization}

The coding sequence of PeuLAC2 was cloned into the vector 35S::pEarleyGate-YFP via Gateway technology (Invitrogen, Germany) with subcellular primers (Table S2). The construct 35S::PeuLAC2-YFP and empty vector 35S::YFP were introduced into $A$. tumefaciens strain GV3101. The constructs were grown in Luria-Bertani (LB) medium with $20 \mu \mathrm{M}$ acetosyringone (AS). When the optical density was $600 \mathrm{~nm}\left(\mathrm{OD}_{600}\right)=0.5$, the cultures were resuspended in injection buffer (liquid MS medium with $10 \mathrm{mM} \mathrm{MgCl} 2,150 \mu \mathrm{M}$ acetosyringone, and $10 \mathrm{mM}$ MES, pH 5.8). Then, resuspended cultures of 35S::PeuLAC2-YFP and 35S::YFP were injected into 3-week-old Nicotiana benthamiana leaves. A confocal laser-scanning microscope (TCSSP8, Leica) was used to observe YFP fluorescence at $488 \mathrm{~nm}$ emission $48 \mathrm{~h}$ after injection. Tissues infected with 35S::YFP were used as negative controls.

\section{Analysis of drought-stress tolerance}

For the drought treatment, the At-OE and At-WT Arabidopsis seedlings were cultured on MS medium. After 5 days, the seedlings were transferred into pots containing equal amounts of soil by weight for 2 weeks with adequate watering. Drought treatment was performed by withholding water for 12-18 days, and wellhydrated plants were used as controls. Watering resumed when the At-WT plants showed a fatal dehydration phenotype. After rewatering for 2 days, the survival rate of the stressed plants was calculated. Three independent experiments were performed. Each independent experiment contained six pots of At-WT and At-OE plants, respectively, and each pot contained nine Arabidopsis seedlings. We then examined water loss and the leaf relative water content (RWC). The rosette leaves of 4-week-old Arabidopsis plants were detached and placed into a glass culture dish at $22^{\circ} \mathrm{C}$ and $30-40 \% \mathrm{RH}$. Then, the weight of the detached rosette leaves was measured every $1 \mathrm{~h}$ for $8 \mathrm{~h}$. Water loss was calculated as the percentage of the leaf fresh weight that was lost. The RWC was assessed using rosette leaves from 2-week-old plants after withholding irrigation for 12 days, and well-hydrated plants were used as controls. Three biological replicates were performed.

We also evaluated the drought resistance of transgenic $P$. alba plants. Five-week-old poplar saplings were transferred into the soil in pots containing equal amounts of soil by weight and grown for 5 months with sufficient watering. Saplings at the same growth stage were subjected to drought stress implemented by withholding water, and 
well-hydrated plants served as controls. After 10 days, signs of leaf wilting and necrosis were observed. After 16 days, the plants were rewatered and maintained under well-watered conditions for 5 days, and their survival rate was calculated. To examine the stem water potential, poplar plants were cultured under well-hydrated and unwatered conditions for 16 days. According to the manufacturer's instructions, the stem water potential was measured with a SAPS II Water Potential System (SEC). Three independent drought resistance experiments were performed. Each independent experiment included nine $\mathrm{Pal}-\mathrm{WT}$ and Pal-OE saplings, respectively.

The proline (PRO), malondialdehyde (MDA), and hydrogen peroxide $\left(\mathrm{H}_{2} \mathrm{O}_{2}\right)$ contents and antioxidant capacities, including the catalase (CAT), superoxide dismutase (SOD) and peroxidase (POD) activities, were measured by spectrophotometry as described in the protocols of the kits from Suzhou Comin Biotechnology (www.cominbio.com). Three biological replicates and three technical replicates were performed.

\section{Determination of SCW thickness and chemical components}

For the determination of SCW thickness, Arabidopsis inflorescence stems were cut into $2-3 \mathrm{~mm}$ segments from $0.5 \mathrm{~cm}$ above the rosette leaves and then fixed for cell wall observation under a transmission electron microscope (TEM; HITACHI H-7650) according to the manufacturer's protocols ${ }^{57}$. ImageJ (https://imagej.nih.gov/ij/) was used to measure the SCW thickness, and at least 30 cells in each of three plants were measured.

The chemical components of the SCW, such as its lignin, cellulose, and noncellulosic polysaccharide contents, were determined using previously described methods ${ }^{58-60}$. Arabidopsis inflorescence stems and 2month-old poplar stem segments between the 2nd and 5 th internodes were harvested, ground into a fine powder in liquid nitrogen, and used to generate alcohol insoluble residue (AIR) for the determination of the lignin, cellulose, and noncellulosic polysaccharide contents. Three biological replicates and three technical replicates were performed.

\section{Cross-sectioning and histological staining}

Arabidopsis inflorescence stems and the fifth internode of stems from 5-month-old P. alba plants were cut into $0.5-1 \mathrm{~cm}$ segments, fixed in FAA solution, and embedded in paraffin. A rotary microtome (RM2235, Leica) was used to section the embedded fragments to a thickness of $12 \mu \mathrm{m}$. The sections were stained with $0.1 \%$ toluidine blue and observed using an optical microscope (Zeiss, Germany). ImageJ software was used to analyze the images to quantify the morphological parameters of the xylem cells, and at least 30 xylem cells in each of three plants were measured.

\section{RNA-seq analysis}

For RNA-seq, water was withheld from 2-week-old PeuLAC2-OE and At-WT Arabidopsis plants for 15 days. The rosette leaves of five independent plants under drought stress and well-watered conditions were collected. Total RNA for RNA-seq was extracted by BIOMARKER (Beijing, China). To trim the reads, we removed adapter sequences, low-quality sequences, low-complexity sequences, and reads with many (>5\%) unknown bases. The TAIR10 Arabidopsis genome was used to align the resultant clean reads with the HISAT2 program (v2.0.4). The gene expression level and fragments per kilobase of transcripts per million fragment mapped (FPKM) values were quantified and calculated using RSEM (v1.2.12). Analysis of the differentially expressed genes (DEGs) was performed using the DESeq2 $\mathrm{R}$ package. Genes with an adjusted $P$-value $<0.05$ and fold change $(\mathrm{FC}) \geq 2(\mid \log 2$ $(\mathrm{FC}) \mid \geq 1$ ) were considered differentially expressed by DESeq2.

Quantitative RT-PCR was performed to examine gene expression levels using TB Green ${ }^{\circledR}$ Premix Ex Taq ${ }^{\mathrm{mm}}$ (TaKaRa) with a real-time PCR detection system (MX3005P, Agilent). Gene expression levels were normalized using ACTIN2 as an internal control. The relative expression levels of the target genes were calculated according to the $2^{-\triangle \triangle C T}$ method $^{61}$. All experiments were performed with five biological replicates and three technical replicates.

\section{Statistical analyses}

Statistical analyses to test the data from all experiments for significant differences were performed using SPSS version 16.0. Data are expressed as the means \pm SDs based on at least three independent biological replicates. Differences were considered significant at $P \leq 0.05$ according to Student's $t$-test.

\section{Acknowledgements \\ This research was supported by the National Natural Science Foundation of China (No. 31870580 and 31470620) and the Supercomputing Center of Lanzhou University. The Core Facility of the School of Life Sciences, Lanzhou University, provided us with the gRT-PCR facilities.}

\section{Author contributions \\ D.W. and J.L. designed the experiments. Z.N., G.L., and H.H. performed the experiments. G.L., H.H. and Q.Z. contributed to the data analyses. Z.N., D.W., and J.L. wrote the manuscript.}

Conflict of interest

The authors declare no competing interests.

Supplementary information The online version contains supplementary material available at https://doi.org/10.1038/s41438-021-00518-x.

Received: 23 July 2020 Revised: 20 December 2020 Accepted: 6 February 2021

Published online: 01 April 2021 


\section{References}

1. Basu, S., Ramegowda, V., Kumar, A. \& Pereira, A. Plant adaptation to drought stress. F1000 Res. 5, 1554 (2016).

2. Ma, J. et al. Genome-wide survey reveals transcriptional differences underlying the contrasting trichome phenotypes of two sister desert poplars. Genes $\mathbf{7}$ 111 (2016).

3. Ullah, A. et al. Phytohormones enhanced drought tolerance in plants: a coping strategy. Environ. Sci. Pollut. Res. 25, 33103-33118 (2018).

4. Hochberg, U. et al. Grapevine acclimation to water deficit: the adjustment of stomatal and hydraulic conductance differs from petiole embolism vulnerability. Planta 245, 1091-1104 (2017).

5. Delzon, S., Douthe, C., Sala, A. \& Cochard, H. Mechanism of water-stress induced. cavitation in conifers: bordered pit structure and function support the hypothesis of seal capillary-seeding. Plant Cell Environ. 33, 2101-2111 (2010).

6. Mot, A. C. \& Silaghi-Dumitrescu, R. Laccases: complex architectures for oneelectron oxidations. Biochemistry 77, 1395-1407 (2012).

7. Yoshida, H. LXIII.-chemistry of lacquer (Urushi). Part I. communication from the chemical society of Tokio. J. Chem. Soc. 43, 472-486 (1883).

8. Wang, J. et al. Lignin engineering through laccase modification: a promising field for energy plant improvement. Biotechnol. Biofuels 8, 145 (2015).

9. Pourcel, $L$. et al. TRANSPARENT TESTA10 encodes a laccase-like enzyme involved in oxidative polymerization of flavonoids in Arabidopsis seed coat. Plant Cell 17, 2966-2980 (2005)

10. Liu, Q., Luo, L., Wang, X., Shen, Z. \& Zheng, L. Comprehensive analysis of rice laccase gene (OSLAC) family and ectopic expression of OSLAC10 enhances tolerance to copper stress in Arabidopsis. Int. J. Mol. Sci. 18, 209 (2017).

11. Berthet, S. et al. Disruption of LACCASE4 and 17 results in tissue-specific alterations to lignification of Arabidopsis thaliana stems. Plant Cell $\mathbf{2 3}$ 1124-1137 (2011).

12. Turlapati, P. V., Kim, K. W., Davin, L. B. \& Lewis, N. G. The laccase multigene family in Arabidopsis thaliana: towards addressing the mystery of their gene function (s). Planta 233, 439-470 (2011).

13. Liang, M., Davis, E., Gardner, D., Cai, X. \& Wu, Y. Involvement of AtLAC15 in lignin synthesis in seeds and in root elongation of Arabidopsis. Planta 224, 1185-1196 (2006)

14. Zhao, Q. et al. LACCASE is necessary and nonredundant with PEROXIDASE for lignin polymerization during vascular development in Arabidopsis. Plant Cell 25, 3976-3987 (2013).

15. Cheng, $X$. et al. Comprehensive genome-wide analysis of the pear (Pyrus bretschneideri) laccase gene (PbLAC) family and functional identification of PbLAC1 involved in lignin biosynthesis. PLOS ONE 14, e0210892 (2019).

16. Ranocha, P. et al. Laccase down-regulation causes alterations in phenolic metabolism and cell wall structure in poplar. Plant Physiol. 129, 145-155 (2002).

17. Lu, S. et al. Ptr-miR397a is a negative regulator of laccase genes affecting lignin content in Populus trichocarpa. Proc. Natl Acad. Sci. USA 110, 10848-10853 (2013).

18. Wang, C. et al. MiR397b regulates both lignin content and seed number in Arabidopsis via modulating a laccase involved in lignin biosynthesis. Plant Biotechnol. J. 12, 1132-1142 (2014)

19. Swetha, C. et al. Major domestication-related phenotypes in indica rice are due to loss of miRNA-mediated laccase silencing. Plant Cell 30, 2649-2662 (2018).

20. Hu, Q. et al. Laccase GhLac1 modulates broad-spectrum biotic stress tolerance via manipulating phenylpropanoid pathway and jasmonic acid synthesis. Plant Physiol. 176, 1808-1823 (2018).

21. Zhang, Y. et al. The cotton laccase gene GhLAC15 enhances Verticillium wilt resistance via an increase in defence-induced lignification and lignin components in the cell walls of plants. Mol. Plant Pathol. 20, 309-322 (2019).

22. Cai, $X$. et al. Mutant identification and characterization of the laccase gene family in Arabidopsis. J. Exp. Bot. 57, 2563-2569 (2006).

23. Cho, H. et al. Overexpression of the OsCh/1 gene, encoding a putative laccase precursor, increases tolerance to drought and salinity stress in transgenic Arabidopsis. Gene 552, 98-105 (2014).

24. Gries, D. et al. Growth and water relations of Tamarixra mosissima and Populus euphratica on Taklamakan desert dunes in relation to depth to a permanent water table. Plant Cell Environ. 26, 725-736 (2003).

25. Ma, T. et al. Genomic insights into salt adaptation in a desert poplar. Nat. Commun. 4, 2797 (2013)
26. Zeng, F., Yan, H. \& Arndt, S. K. Leaf and whole tree adaptations to mild salinity in field grown Populus euphratica. Tree Physiol. 29, 1237-1246 (2009).

27. $\mathrm{Ma}, \mathrm{X}$. et al. Genome-wide identification of TCP family transcription factors from Populus euphratica and their involvement in leaf shape regulation. Sci. Rep. 6, 32795 (2016).

28. Thevs, N. et al. Structure and wood biomass of near-natural floodplain forests along the Central Asian rivers Tarim and Amu Darya. Forestry 85, 193-202 (2011).

29. Eckert, C. et al. What makes the wood? Exploring the molecular mechanisms of xylem acclimation in hardwoods to an ever-changing. Environ. For. 10, 358 (2019).

30. Hoson, T. \& Wakabayashi, K. Role of the plant cell wall in gravity resistance. Phytochemistry 112, 84-90 (2015).

31. Underwood, W. The plant cell wall: a dynamic barrier against pathogen invasion. Front. Plant Sci. 3, 85 (2012)

32. Bai, $X$. et al. A novel gene coding $Y$-aminobutyric acid transporter (GAT) may improve the tolerance of Populus euphratica to adverse environments. Front Plant Sci. 10, 1083 (2019).

33. Ahuja, I. A., de Vos, R. C. H., Bones, A. M. \& Hall, R. D. Plant molecular stress responses face climate change. Trends Plant Sci. 15, 664-674 (2010).

34. $\mathrm{Yu}, \mathrm{L}$. et al. Tissue-specific transcriptome analysis reveals multiple responses to salt stress in Populuseuphratica seedlings. Genes 8, 372 (2017).

35. Yang, Z. PAML 4: phylogenetic analysis by maximum likelihood. Mol. Biol. Evol. 24, 1586-1591 (2007)

36. Choat, B. et al. Global convergence in the vulnerability of forests to drought. Nature 491, 752-755 (2012).

37. Nowak, R. Entering the postgenome era. Science 270, 368-371 (1995).

38. Wagner, A. Redundant gene functions and natural selection. J. Evol. Biol. 12, 1-16 (1999).

39. Kafri, R., Springer, M. \& Pilpel, Y. Genetic redundancy: new tricks for old genes Cell 136, 389-392 (2009).

40. Mittler, R., Vanderauwera, S. \& Gollery, M. \& Van Breusegem, F. Reactive oxygen gene network of plants. Trends Plant Sci. 9, 490-498 (2004)

41. Green, F. III \& Highley, T. L. Mechanism of brown-rot decay: paradigm or paradox. Int. Biodeterior. Biodegrad. 39, 113-124 (1997).

42. Fisher, J. B., Goldstein, G., Jones, T. J. \& Cordell, S. Wood vessel diameter is related to elevation and genotype in the Hawaiian tree Metrosideros polymorpha (Myrtaceae). Am. J. Bot. 94, 709-715 (2007).

43. Skirycz, A. \& Inzé, D. More from less: Plant growth under limited water. Curr. Opin. Biotechnol. 21, 197-203 (2010).

44. Awasthi, M., Jaiswal, N., Singh, S., Pandey, V. P. \& Dwivedi, U. N. Molecular docking and dynamics simulation analyses unraveling the differential enzymatic catalysis by plant and fungal laccases with respect to lignin biosynthesis and degradation. J. Biomol. Struct. Dyn. 33, 1835-1849 (2015).

45. Madhavi, V. \& Lele, S. S. Laccase: properties and applications. BioResources 4 1694-1717 (2009)

46. Hakulinen, N. et al. Crystal structure of a laccase from Melanocarpus albomyces with an intact trinuclear copper site. Nat. Struct. Biol. 9, 601-605 (2002).

47. Messerschmidt, A. \& Huber, R. The blue oxidases, ascorbate oxidase, laccase and ceruloplasmin modelling and structural relationships. Eur. J. Biochem. 187, 341-352 (1990)

48. Hashimoto-Sugimoto, M. et al. A Munc13-like protein in Arabidopsis mediates $\mathrm{H}^{+}$-ATPase translocation that is essential for stomatal responses. Nat. Commun 4, 2215 (2013)

49. Li, L. et al. The rice OsDUF810 family: OsDUF810. 7 May be involved in the tolerance to salt and drought. Mol. Biol. 52, 489-496 (2018).

50. Tognetti, V. B. et al. Perturbation of indole-3-butyric acid homeostasis by the UDP-glucosyltransferase UGT74E2 modulates Arabidopsis architecture andwater stress tolerance. Plant Cell 22, 2660-2679 (2010).

51. Somerville, C. Cellulose synthesis in higher plants. Annu. Rev. Cell Dev. Biol. 22 53-78 (2006).

52. Olsen, A. N., Ernst, H. A., Leggio, L. L. \& Skriver, K. NAC transcription factors: structurally distinct, functionally diverse. Trends Plant Sci. 10, 79-87 (2005).

53. Yamaguchi, M. \& Demura, T. Transcriptional regulation of secondary wall formation controlled by NAC domain proteins. Plant Biotechnol. 27, 237-242 (2010).

54. Clough, S. J. \& Bent, A. F. Floral dip: a simplified method for Agrobacteriummediated transformation of Arabidopsis thaliana. Plant J. 16, 735-743 (1998).

55. Jia, Z., Sun, Y., Yuan, L., Tian, Q. \& Luo, K. The chitinase gene (Bbchit1) from Beauveria bassiana enhances resistance to Cytospora chrysosperma in Populus tomentosa Carr. Biotechnol. Lett. 32, 1325-1332 (2010). 
56. Ma, J. et al. Genome sequence and genetic transformation of a widely distributed and cultivated poplar. Plant Biotechnol. J. 17, 451-460 (2019).

57. Zhao, Y., Sun, J., Xu, P., Zhang, R. \& Li, L. Intron-mediated alternative splicing of WOOD-ASSOCIATED NAC TRANSCRIPTION FACTOR1B regulates cell wall thickening during fiber development in Populus species. Plant Physiol. 164, 765-776 (2014)

58. Foster, C. E., Martin, T. M. \& Pauly, M. Comprehensive compositional analysis of plant cell walls (lignocellulosic biomass) part I: lignin. J. Vis. Exp. 37, e1745 (2010)
59. Foster, C. E., Martin, T. M. \& Pauly, M. Comprehensive compositional analysis of plant cell walls (lignocellulosic biomass) part II: carbohydrates. J. Vis.Exp. 37, e1837 (2010).

60. Zhang, Q. et al. Blue light regulates secondary cell wall thickening via MYC2 MYC4 activation of the NST1-directed transcriptional network in Arabidopsis. Plant Cell 30, 2512-2528 (2018).

61. Schmittgen, T. D. \& Livak, K. J. Analyzing real-time PCR data by the comparative $C_{T}$ method. Nat. Protoc. 3, 1104 (2008). 